\title{
¿QUIÉN ES QUIÉN? DIRECTRICES DE USO DE UNA CLAVE DICOTÓMICA PARA LA IDENTIFICACIÓN DE ARTRÓPODOS EN EDUCACIÓN PRIMARIA
}

Rosa Gálvez Esteban ${ }^{1}$

Universidad Autónoma de Madrid

Recibido 29/04/2021 Aceptado 31/05/2021

\begin{abstract}
$Z_{\text {Iy }}$ El objetivo de este trabajo es aportar material didáctico original diseñado para clasificar artrópodos, así como las directrices metodológicas oportunas para implementar su uso en Educación Primaria. Es muy interesante trabajar con artrópodos como modelo animal en la etapa educativa de Educación Primaria por la elevada diversidad de modelos anatómicos que presentan y por la facilidad de observarlos en su entorno. Además, la adaptación de herramientas reales que utilizan los científicos como son las claves dicotómicas, y el desarrollo de destrezas propias del trabajo científico como son la observación o la clasificación, permiten el acercamiento del alumnado al quehacer científico, y es además una vía que permite mejorar la comprensión del medio natural a partir de situaciones contextualizadas.
\end{abstract}

5 The aim of this work is to provide original teaching material designed to classify arthropods, as well as the appropriate methodological guidelines to implement its use in Primary Education. It is very interesting to work with arthropods as animal models in Primary Education due to the great diversity of anatomical models they present and the ease of observing them in their environment. Moreover, the adaptation of real tools used by scientists, such as dichotomous keys, and the development of skills and abilities inherent to scientific work, such as observation and classification, allow pupils to get closer to scientific work, and is also a way of improving their understanding of the natural environment through contextualised situations.

DOI

https://doi.org/10.15366/didacticas2021.24.005

PALABRAS CLAVE

Clave dicotómica; Artrópodos; Clasificación; Educación Primaria. 


\section{INTRODUCCIÓN}

Hoy en día, vivimos en una sociedad alejada del entorno natural, lo que dificulta la comprensión de éste y sus componentes (Clemens, 2004). Para suplir esa carencia, se hace imprescindible trabajar en el aula con organismos reales, brindando oportunidades al alumnado para que puedan observarlos por sí mismos y aumentar así su interés y motivación (Gálvez y col., 2020). El uso de secuencias didácticas a partir de situaciones contextualizadas en las que prime la observación directa es crucial para mejorar la comprensión del medio natural a través de un aprendizaje significativo y transferible que ayude a reconocer la necesidad de proteger el entorno y conservar la diversidad biológica (Álvarez y col., 2017; BOCM, 2014).

La enseñanza de la biodiversidad, desde su perspectiva tangible, permite reconocer las características diagnósticas de los seres vivos con el fin de poder distinguir unas especies de otras, así como los grupos a los que pertenecen (Álvarez y col., 2017; Torralba y col., 2017). Dichas características permiten clasificar en grupos a aquellos organismos que compartan caracteres, y que, a su vez, puedan diferenciarse de los miembros de otros grupos (Vilches y col., 2012).

El objetivo de este trabajo es aportar material didáctico original diseñado para clasificar artrópodos, y las directrices metodológicas oportunas para su uso en Educación Primaria.

\section{¿QUÉ ES UNA CLAVE DICOTÓMICA Y CÓMO SE UTILIZA?}

Como ha sido descrito previamente por varios autores, es crucial comprender qué es y cómo se utiliza una clave dicotómica para implementar su uso en el entorno educativo (Álvarez y col, 2017; Gálvez y Melero-Alcibar, 2019):

Una clave dicotómica es una herramienta que permite clasificar tanto seres vivos como materia inerte (rocas, minerales, etc.) (Álvarez y col, 2017; Martín Del Pozo y Galán Martín, 2012) mediante una serie de dilemas encadenados (SI/NO) basados en la presencia o no de caracteres específicos en los elementos a identificar. Constan de dos proposiciones que se excluyen mutuamente. Observando detenidamente el ejemplar, hay que admitir una y rechazar la otra. Tras la elección de uno u otro se determinará el camino a seguir, hasta llegar a su precisa determinación. Es importante comprender el significado de los términos que aparezcan en las proposiciones antes de seguir avanzando porque nos evitará llegar a un resultado erróneo. Si, aun así, al llegar a un dilema observamos que ninguna de las características descritas en las dos proposiciones coincide con las de nuestro ejemplar, significa que se ha seguido un camino equivocado. Entonces, hay que retroceder en la clave hasta el dilema en el que no se eligió correctamente la proposición, o bien, empezar de nuevo. 


\section{¿POR QUÉ USAR CLAVES DICOTÓMICAS EN EDUCACIÓN PRIMARIA?}

Es objeto de estudio en el ámbito de la asignatura troncal de Ciencias de la Naturaleza, comprender y describir los elementos que forman parte de la naturaleza. De hecho, en el Real Decreto 126/2014 de 28 de febrero por el que se establece el currículo básico de la Educación Primaria, se señala la importancia de la utilización de guías en la identificación de animales y plantas observando sus características. De forma más concreta, en el Decreto 89/2014 de 24 de julio del Consejo de Gobierno por el que se establece para la Comunidad de Madrid el Currículo de la Educación Primaria, esos contenidos se enmarcan en tercer curso de la etapa de Educación Primaria. Es esencial que la enseñanza de los contenidos de esta área se haga de forma eminentemente práctica (BOCM, 2014), por eso es de gran importancia que en el aula se trabaje de forma directa con colecciones de seres vivos o con recursos en colecciones virtuales digitalizadas (Torralba y col., 2017; Gálvez y Melero-Alcibar, 2019).

Los criterios y estándares de evaluación suponen una formulación evaluable de las capacidades expresadas en los objetivos generales de la Educación Primaria, asociadas a los contenidos fundamentales de esta área, y muestran las competencias que el alumnado debe adquirir (BOE, 2014). Los estándares de aprendizaje evaluables que se establecen en el currículo básico relacionados con el uso de claves dicotómicas son:

- Observa directa e indirectamente, identifica características, reconoce y clasifica animales y plantas

- Utiliza guías en la identificación de animales y plantas

Asimismo, existen estándares de aprendizaje evaluables que se establecen en el currículo básico en el ámbito de otra asignatura troncal, Ciencias Sociales, donde se contempla la clasificación de minerales (BOE, 2014):

- Identifica y explica las diferencias entre rocas y minerales, describe sus usos y utilidades, clasificando algunos minerales según sus propiedades.

En lo que se refiere a los contenidos procedimentales, los relacionados con el «saber hacer» teórico y práctico, los/las alumnos/as han de iniciarse en conocer y utilizar algunas de las estrategias y técnicas habituales en la actividad científica (BOE, 2014). De forma más concreta, podemos aseverar que se trabajan contenidos procedimentales basados en destrezas básicas como la observación (identificación de propiedades observables) o la clasificación (utilización de criterios de clasificación o claves) (Pro, 2013). Asimismo, el uso de guías de identificación puede promover la curiosidad, el interés y el respeto hacia la naturaleza desarrollando actitudes y valores relacionados con el «saber ser» (BOCM, 2014). Ambos contenidos, tanto los procedimentales como los actitudinales, se organizan alrededor de conceptos fundamentales contemplados en el currículo básico de Educación Primaria: "los seres vivos". 
Esta propuesta de aula presenta un enfoque que permite al alumnado desarrollar las siguientes competencias clave generando cambios favorables en el aprendizaje del alumnado: a) Comunicación lingüística, b) Competencias básicas en ciencia y tecnología y c) Aprender a aprender (Orden ECD/65, 2015).

Además, como afirman Álvarez y col. (2017), es importante la utilización de criterios de clasificación en el aula ya que su uso inadecuado estableciendo, por ejemplo, clasificaciones artificiales en animales presentando y describiendo sus características de forma aislada (animales domésticos, de granja o salvajes), se muestra resistente al cambio y puede derivar en ideas alternativas persistentes en sucesivas etapas educativas (Chyleńska y Rybska,. 2018).

\section{CONFECCIÓN DE LA CLAVE DICOTÓMICA DE ARTRÓPODOS}

Es muy interesante trabajar con artrópodos como modelo animal en la etapa educativa de Educación Primaria por la elevada diversidad de modelos anatómicos que presentan y por la facilidad de observarlos en su entorno (Ribera y col., 2015). Tradicionalmente, el uso de claves dicotómicas ha estado limitado al ámbito científico, pero si están acompañadas por dibujos explicativos, como en la clave dicotómica que se presenta a continuación, éstos son de gran utilidad y pueden ser empleadas fácilmente por alumnos de E. primaria (Vilches y col., 2012).

El uso de la clave dicotómica implica conocer las características distintivas de los organismos de cada grupo de estudio. En esta clave (Fig.1), la determinación se precisará a nivel de clase en el caso de arácnidos, crustáceos y miriápodos; y a nivel de orden en el caso de los insectos (14 órdenes en total). Para la construcción de la clave se han tenido en cuenta las siguientes características de los artrópodos con el fin de elaborar un sistema de dos opciones:

- patas (número, tamaño con relación al cuerpo, adaptaciones anatómicas)

- $\quad$ antenas (presencia o ausencia)

- alas (presencia o ausencia, número, consistencia, estructura, venación, tamaño con relación al cuerpo)

- morfología general del cuerpo (presencia o ausencia de las siguientes adaptaciones anatómicas: pinzas al final del cuerpo, cintura, cuerpo aplastado)

Figura 1. Clave dicotómica de artrópodos [página siguiente] » 


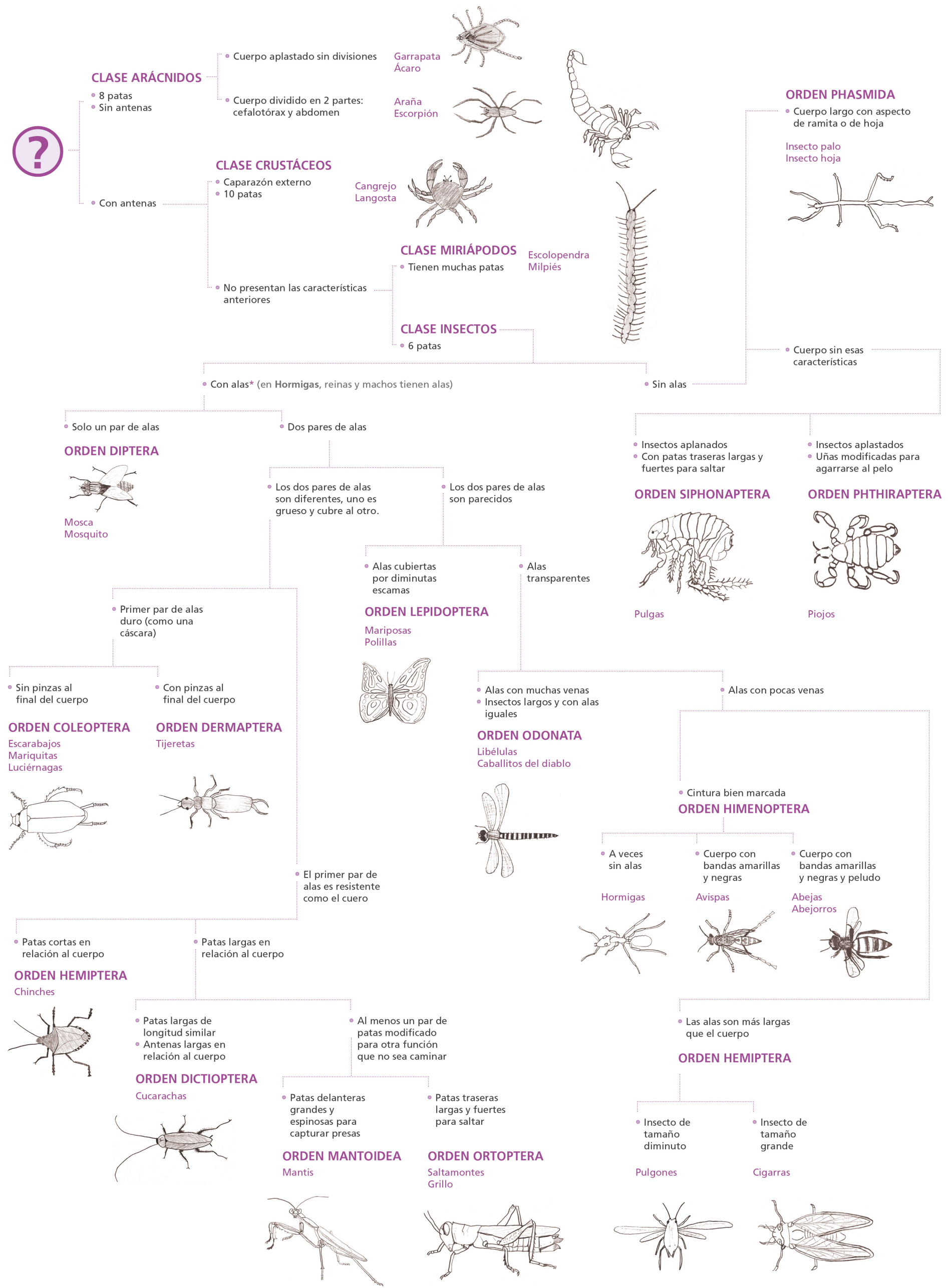


Las dos primeras características propuestas, número de patas y presencia o ausencia de antenas, generan el dilema número 1:

1a. 8 patas y sin antenas Clase Arácnidos

1b. con antenas.

Para continuar el camino número 2, se vuelve a utilizar el número de patas junto con la presencia o ausencia de caparazón externo:

2a. 10 patas $^{1}$ y con caparazón externo

Clase Crustáceos

2b. con antenas

El dilema número 3 se decide de nuevo por el número de patas a través de dos posibilida des:

3a. muchas patas Clase Miriápodos

3b. seis patas. Clase Insectos [4]

La clase insectos es muy diversa y por eso a partir de este punto las agrupaciones serán órdenes. En el dilema cuarto se observará la presencia o ausencia de alas:

4a. con alas

4b. sin alas.

Si continuamos por el dilema 5, se decidirá la opción adecuada según si el ejemplar presenta uno o dos pares de alas:

5a. un par de alas visibles ${ }^{3}$ Orden Diptera

5b. dos pares de alas.

En el número 6, para proseguir en la identificación, hay que observar la estructura del primer par de alas respecto al segundo:

6a. primer par de alas es más grueso y cubre al otro par de alas.

6b. los dos pares de alas con estructura similar.

1 Con el objetivo de simplificar la diversidad de animales que presenta la clase de los crustáceos, solo se contemplaron los decápodos por ser el grupo más conocidos entre los niños y niñas.

2 En el caso de las hormigas hay que ir por esta vía ya que en las castas de reinas y machos sí las presentan durante el vuelo nupcial.

3 En el caso de los dípteros existen un par de alas vestigiales denominadas balancines, es por eso que se especifica en este paso que las alas sean "visibles". 
En el paso 7 se diferencian los ejemplares según la consistencia del primer par de alas:

7a. primer par de alas duro como una cáscara. [8]

7b. primer par de alas resistente como el cuero

El dilema octavo es definitivo, y se decide según la anatomía de la parte final del abdomen, concretamente según si el ejemplar presenta o no pinzas:

8a. sin pinzas en el extremo posterior del cuerpo Orden Coleoptera

8b. con pinzas en el extremo posterior del cuerpo Orden Dermaptera

El dilema noveno consiste en dilucidar si las patas son cortas o largas con relación al tamaño del cuerpo:

9a. patas cortas con relación al cuerpo Orden Hemiptera

9b. patas largas con relación al cuerpo.

En el paso 10 se analiza si las patas son de una longitud similar o si hay al menos un par de patas están adaptadas a realizar una función que no sea caminar:

10a. patas de longitud similar Orden Dictioptera

10b. al menos un par de patas con otra función que no sea caminar

El dilema 11 es definitivo y se resuelve según si es el primer par de patas o el último el que está modificado en realizar una función concreta:

11a. patas anteriores grandes y espinosas para capturar presas. Orden Mantoidea

11b. patas posteriores largas y robustas adaptadas al salto Orden Ortoptera

Si retomamos el dilema 12 que provenía del dilema sexto, en este caso nos tendremos que fijar en la estructura de las alas:

12a. alas cubiertas por diminutas escamas Orden Lepidoptera

12b. alas transparentes

En el número 13 nos tenemos que fijar en el criterio venación de las alas:

13a. alas con muchas venas Orden Odonata 13b. alas con pocas venas.

El dilema 14 es definitivo y se resuelve según la morfología del cuerpo del ejemplar, si tiene cintura o si el cuerpo es más corto que la longitud de las alas: 
14a. cintura bien marcada.

Orden Himenoptera

14b. cuerpo más corto que la longitud de las alas

Orden Hemiptera

Si retomamos el dilema 15 que provenía del dilema cuarto, en este caso nos tendremos que fijar en la morfología del cuerpo:

15a. cuerpo alargado con aspecto de ramita o de hoja Orden Phasmida

15b. cuerpo sin esas características.

Para finalizar, el dilema 16 determina si los ejemplares presentan una morfología general del cuerpo con aplastamiento lateral o dorsal:

16a. cuerpo aplastado lateralmente Orden Siphonaptera

16b. cuerpo aplastado dorsalmente. . Orden Phthiraptera

\section{ORIENTACIONES METODOLÓGICAS PARA EL USO DE LA CLAVE DICOTÓMICA EN EL AULA}

Es aconsejable que antes de comenzar a trabajar con la clave dicotómica, se trabajen las características anatómicas que presentan los cuatro grandes grupos de artrópodos: arácnidos, crustáceos, miriápodos e insectos (Tabla 1).

\begin{tabular}{|c|c|c|c|c|}
\hline Grupo & INSECTOS & ARÁCNIDOS & MIRIÁPODOS & CRUSTÁCEOS \\
\hline $\begin{array}{c}\text { Número de } \\
\text { patas }\end{array}$ & 6 & 8 & $\begin{array}{c}\text { Variable, general- } \\
\text { mente entre } 34 \text { y } \\
400 \text { patas }\end{array}$ & $\begin{array}{c}\text { Variable, entre } 3 \text { y } \\
14 \text { pares patas }\end{array}$ \\
\hline $\begin{array}{c}\text { Divisiones } \\
\text { del cuerpo }\end{array}$ & $\begin{array}{c}\text { Cabeza, tórax y ab- } \\
\text { domen }\end{array}$ & $\begin{array}{c}\text { Cefalotórax y abdo- } \\
\text { men }\end{array}$ & Cabeza y tronco & $\begin{array}{c}\text { Cefalotórax y abdo- } \\
\text { men }\end{array}$ \\
\hline Antenas & 2 antenas & No tienen antenas & 2 antenas & 2 pares de antenas \\
\hline Alas & $\begin{array}{c}2 \text { pares de alas, } 1 \\
\text { par de alas visibles } \\
\text { o sin alas }\end{array}$ & No tienen alas & No tienen alas & No tienen alas \\
\hline Ojos & 2 compuestos & Varios simples & 2 simples & 2 compuestos \\
\hline
\end{tabular}

Tabla 1. Cuadro sinóptico comparativo de los modelos anatómicos de los principales grupos de artrópodos. 
Una opción muy visual que puede ayudar en la comprensión de las características que presenta cada uno de los grupos, es el uso de maquetas de cada modelo anatómico (Fig. 2). También resulta muy interesante y motivador la proyección del video titulado “¿Es la araña un insecto? - El rap de los arácnidos” (Pequetiempo, 2012). En este video se explican las diferencias entre arácnidos e insectos de forma didáctica.
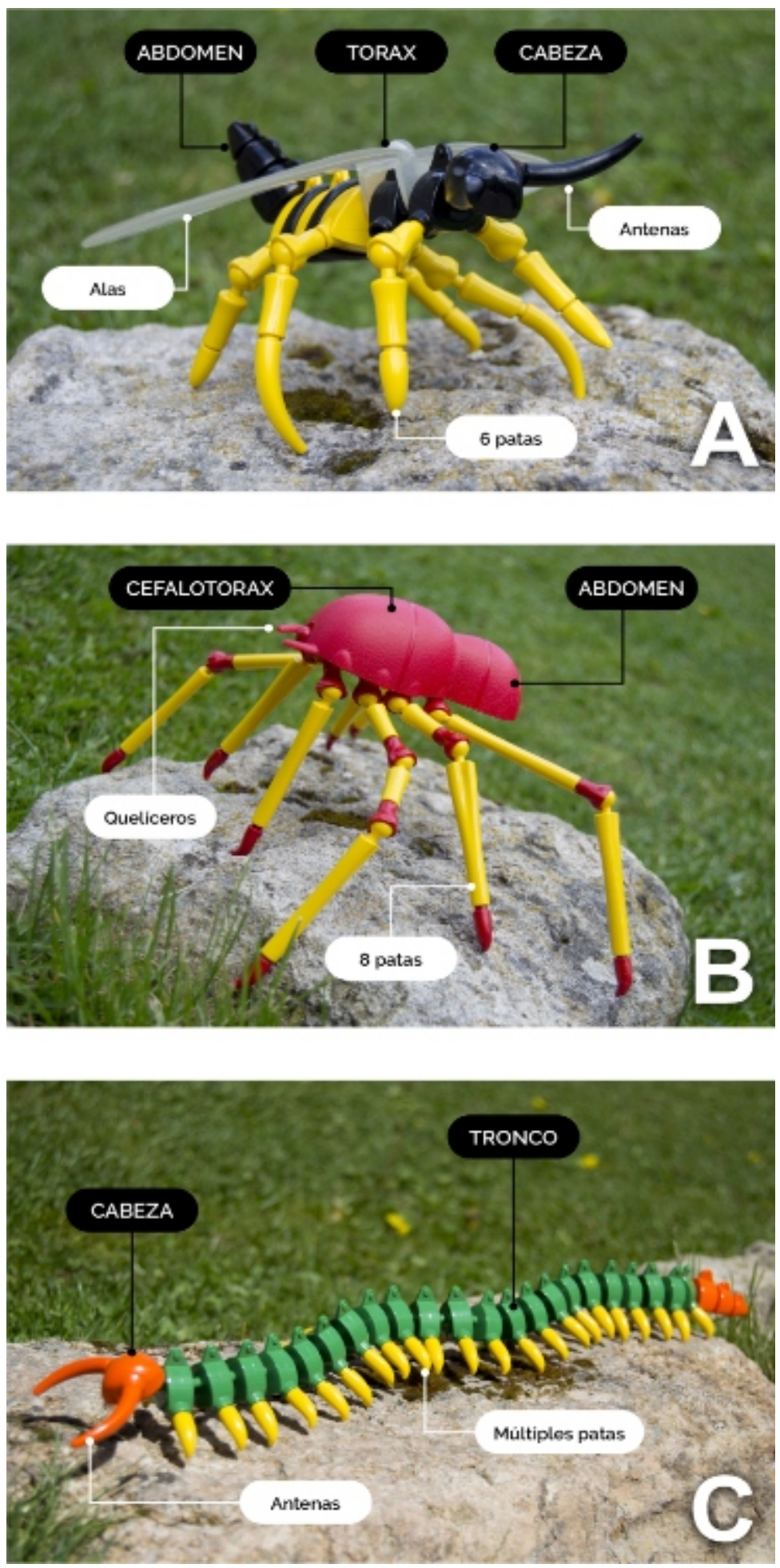

Figura 2. Maquetas de los modelos anatómicos de diferentes grupos de artrópodos: A. insectos; B. arácnidos y C. miriápodos. 
Otro recurso TIC muy interesante es el catálogo de 23 insectos que ofrece Google para visualizarlos en 3D en realidad aumentada. La lista es muy representativa, hay escarabajos, mariposas, saltamontes, libélulas, moscas... Lo primero que necesitamos para poder visualizar estos animales es disponer de un dispositivo compatible que incluye Android, iPhone y iPad. Con el dispositivo compatible, bastará con buscar el animal en el buscador de Google y escoger visualizarlo en 3D (Fig. 3).

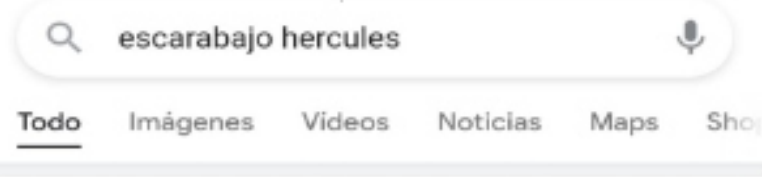

\section{Dynastes hercules}

Insecto

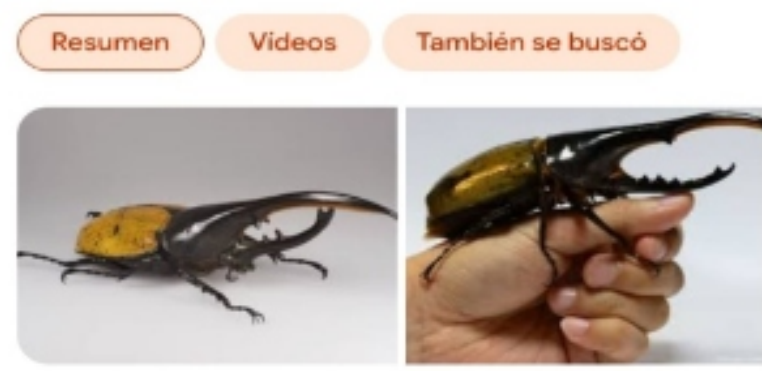

El escarabajo hércules es una especie de coleóptero escarabeido, uno de los escarabajos rinoceronte. Habita en los bosques tropicales, selvas y bosques ecuatoriales de América Central y del Sur. Wikipedia

Nombre cientifico: Dynastes hercules

Observa un ejemplar de escarabajo...

$$
\text { '๑)' Ver en 3D }
$$

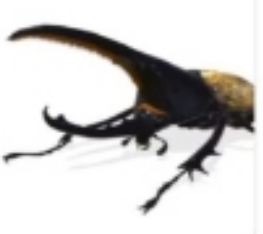

Especie: D. hercules; Linnaeus, 1758

Figura 3. Captura de pantalla del buscador de Google de uno de los 23 insectos del catálogo, el Escarabajo Hércules.

Una vez trabajadas las características más importantes de cada grupo con los recursos anteriormente expuestos, los alumnos y alumnas ya pueden trabajar con la clave dicotómica. El objetivo que tiene que alcanzar el alumnado consiste en escoger de forma adecuada los criterios de clasificación para catalogar ejemplares reales en los diferentes grupos de artrópodos propuestos en una clave dicotómica diseñada ad hoc.

Para realizar una propuesta concreta e implementarla en el aula en tercero de E. Primaria, se recomienda el uso de colecciones de artrópodos conservados en resina plástica por ser un material muy manejable (Fig. 4). También es interesante manejar ejemplares disecados de colecciones, pero tienen el inconveniente de que son más frágiles y difíciles de conseguir (Fig. 5). El uso de estos recursos acompañado del uso de lupas binoculares es un complemento adecuado para observar en detalle estructuras de los ejemplares observados tales como uñas, antenas, escamas de las alas de mariposas, aparato bucal... (Fig. 6). Con este trabajo se plantea el uso de secuencias didácticas en las que se pone en valor el uso de claves dicotómicas usando colecciones de artrópodos, pero incorporando además el uso de recursos innovadores que promuevan un aprendizaje significativo. 


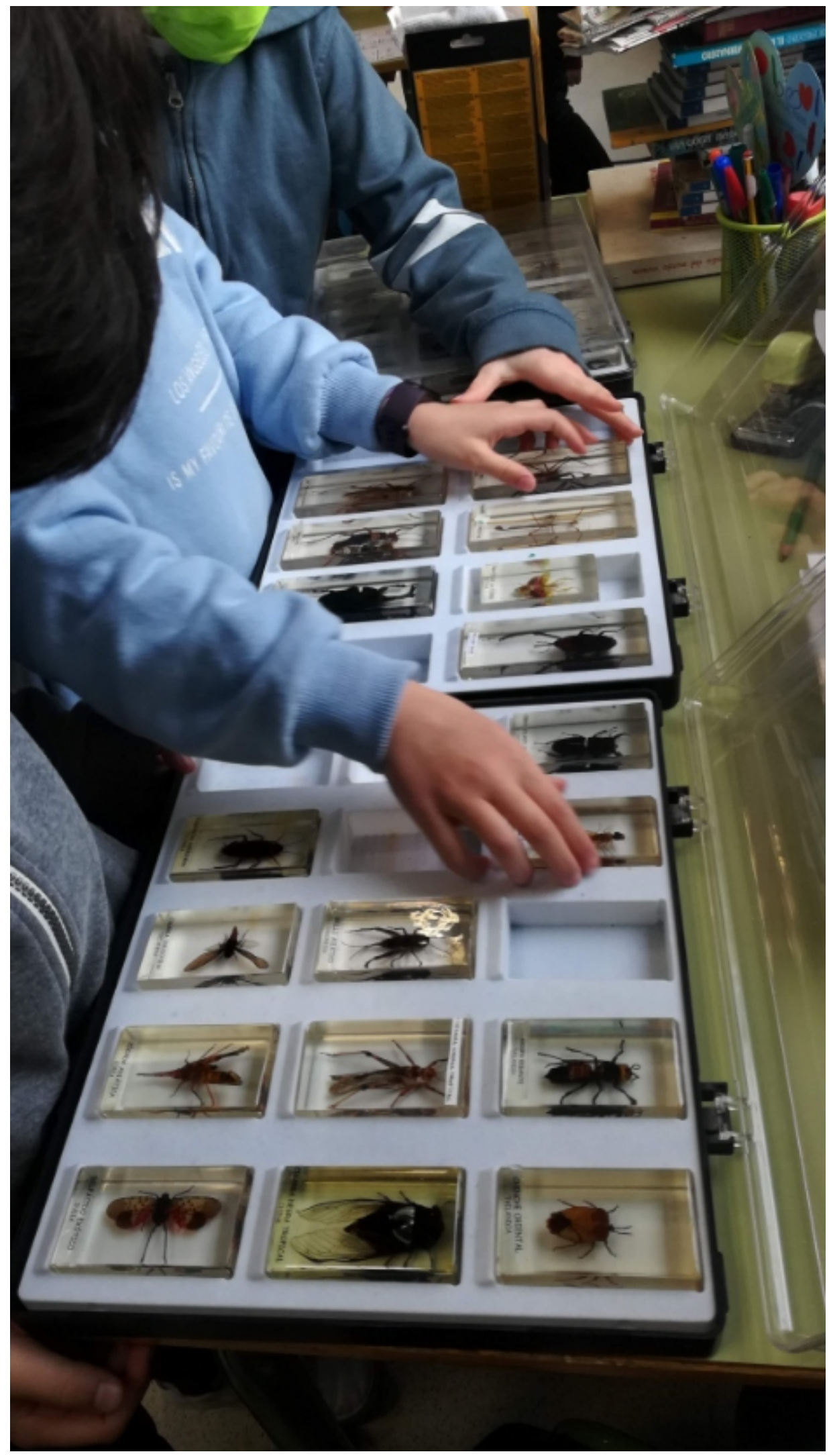

Figura 4. Uso de colecciones de artrópodos conservados en resina plástica en el aula. 


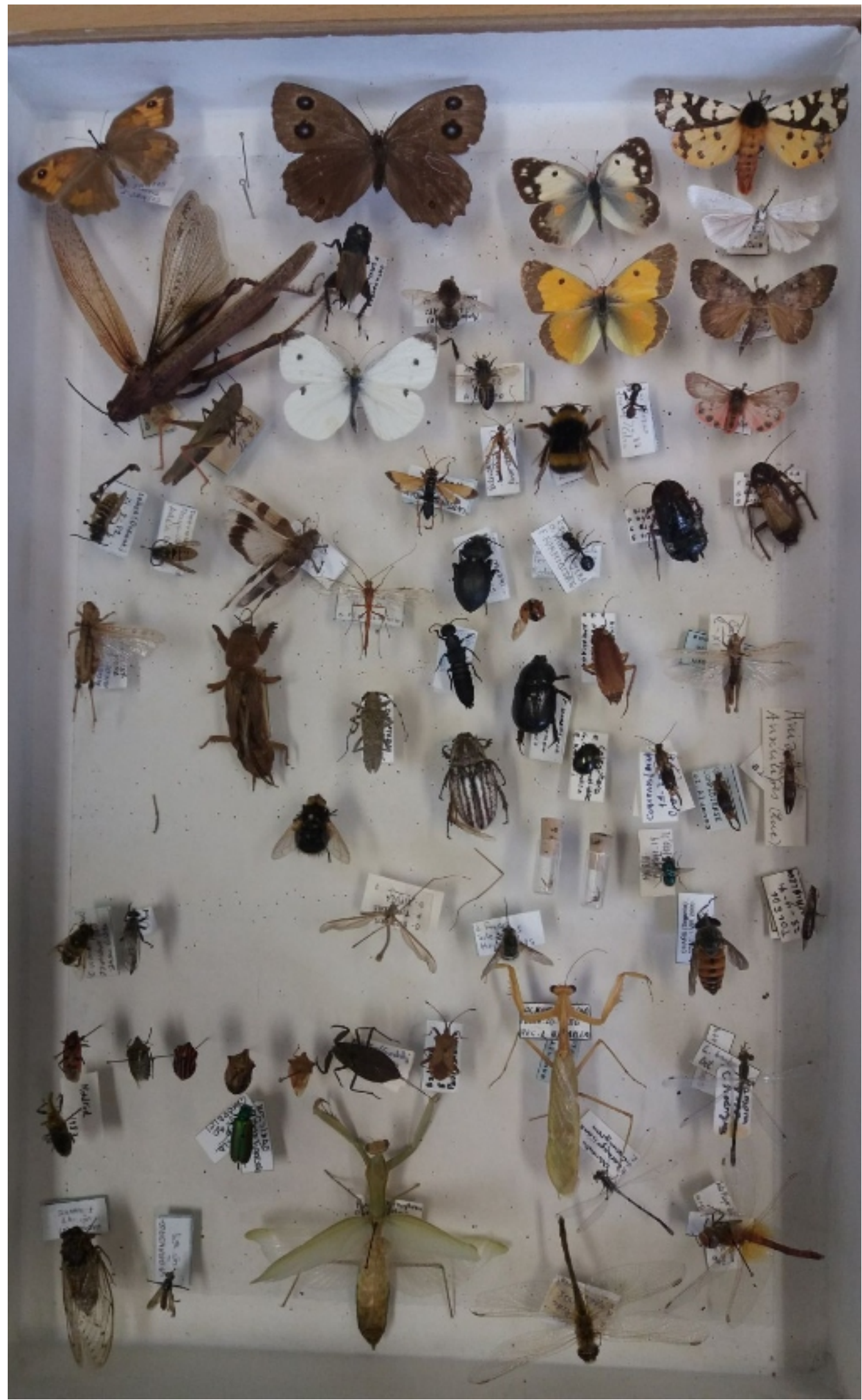

Figura 5. Colección de artrópodos disecados 


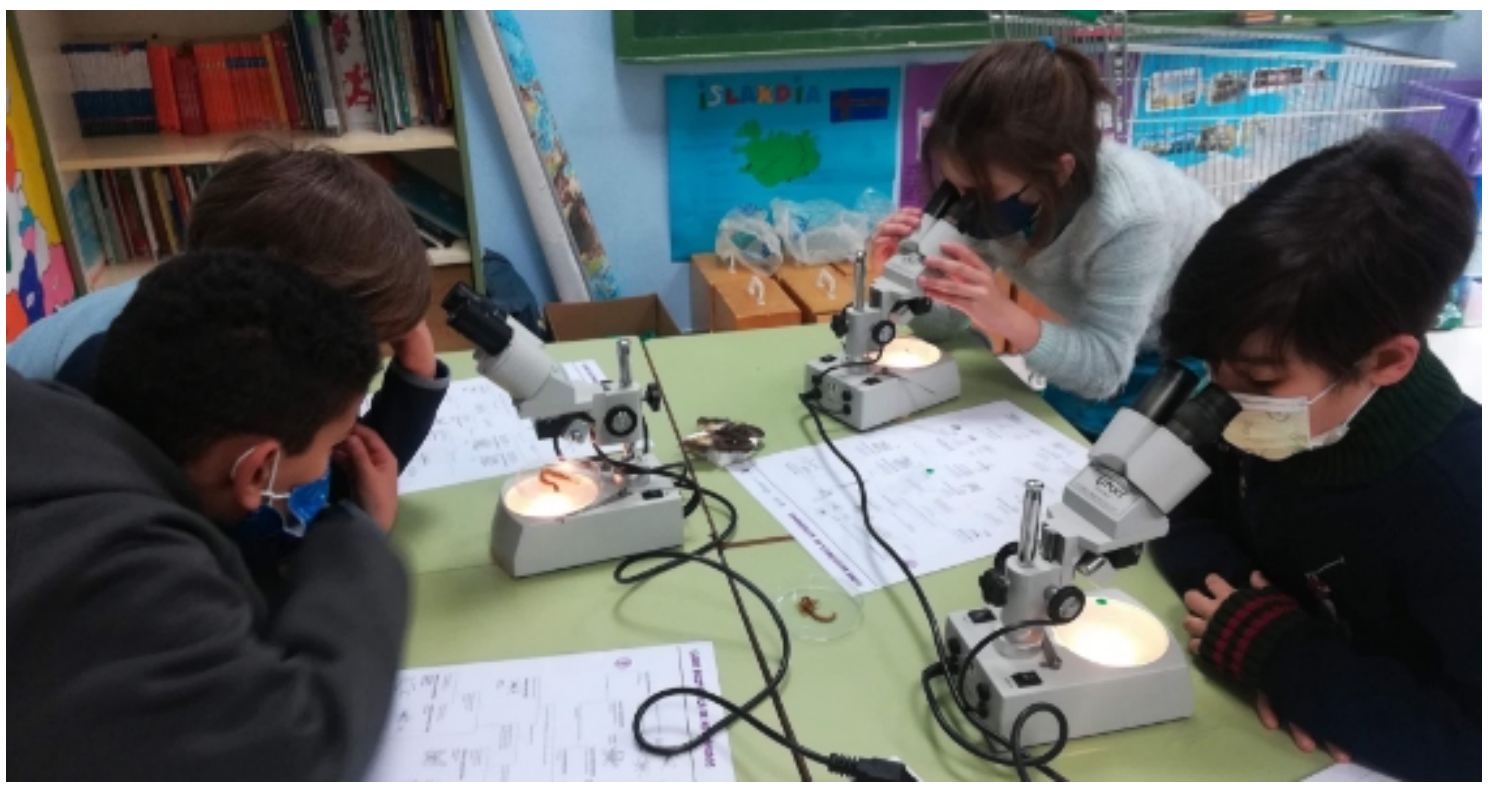

Figura 6. Observación de artrópodos bajo lupa binocular en el aula

\section{REFLEXIONES FINALES}

La clave dicotómica es una herramienta de gran valor científico-didáctico para desarrollar desde la infancia la competencia científica. Cabe destacar que el uso de claves dicotómicas se puede trabajar además de en Educación Primaria, en el ámbito de la Educación Infantil (Gálvez y Melero-Alcibar, 2019; López Carrillo y Cruz Vicente, 2016) y de la Educación Secundaria (Mestres Izquierdo y Torres García, 2008; Álvarez y col., 2017), y permite el estudio de las características básicas de los elementos, su clasificación, así como el estudio de la diversidad.

La adaptación de herramientas reales como las claves dicotómicas que utilizan los científicos permite el acercamiento del alumnado al quehacer científico, y es además una vía que permite enriquecer la forma de enseñar y aprender en el aula desde una mirada integral. De hecho, además de todo lo mencionado anteriormente, con este tipo de propuestas también se promueve el desarrollo de habilidades y destrezas propias del trabajo científico como la observación o la clasificación (Pro, 2013; Vilches y col, 2013). La implementación de este tipo de secuencias didácticas, unido a la utilización de herramientas propias de contextos modernos, permitirá la revalorización de la práctica docente.

\section{AgRADECIMIENTOS}

A Marco Barón Gálvez que en el momento que diseñó los dibujos que acompañan a la clave dicotómica cursaba $4^{\circ}$ de Educación Primaria en el CEIPSO El Encinar de Torrelodones. A Jorge Barón González de Suso por maquetar la clave dicotómica. 


\section{REFERENCIAS}

ÁlVAREZ, J. A., OLIVEROS, C. y DOMÉNECH-CASAL, J. (2017). Diseño y evaluación de una actividad de transferencia entre contextos para aprender las claves dicotómicas y la clasificación de los seres vivos. Revista Electrónica de Enseñanza de las Ciencias, 16, pp.362-384

BOE (2014). Real Decreto 126/2014, de 28 de febrero, por el que se establece el currículo básico de la Educación Primaria. Boletín Oficial del Estado. Madrid, 28 de febrero de 2014, núm. 52, pp.19349-19420.

BOCM (2014). Decreto 89/2014, de 24 de julio, del Consejo de Gobierno, por el que se establece para la Comunidad de Madrid el Currículo de la Educación Primaria. Boletín oficial de la Comunidad de Madrid. Madrid, 24 de julio de 2014, núm. 175, pp.10-89.

CHYLEŃSKA, Z.A. y RYBSKA E. (2018). Understanding Students Ideas about Animal Classification. EURASIA Journal of Mathematics, Science and Technology Education, 14, pp.2145-2155.

CLEMENTS R. (2004). An investigation of the status of outdoor play. Contemporary Issues in Early Childhood, 5, pp.68-80.

Orden ECD/65 (2015). Orden por la que se describen las relaciones entre las competencias, los contenidos y los criterios de evaluación de la educación primaria, la educación secundaria obligatoria y el bachillerato. Boletín Oficial del Estado. Madrid, 29 de enero de 2015, núm. 25, pp. 6986 a 7003.

GÁLVEZ ESTEBAN, R. y MELERO-ALCÍBAR, R. (2019). Viaje al mundo de los animales más pequeños: los artrópodos como recurso educativo en la formación de maestros de Educación Infantil. Educación y Futuro: Revista de investigación aplicada y experiencias educativas, 40, pp.49-72.

GÁLVEZ, R., BRAVO, B. y PÉREZ, J. M. (2020). Ants as an Experiential Learning Strategy in Preschool Teacher Training. En: Interdisciplinary Approaches Toward Enhancing Teacher Education, pp. 134-154. IGI Global.

LÓPEZ CARILLO, M. D. y CRUZ VICENTE, O. DE LA. (2016). Colecciones y claves dicotómicas. Clasificar e identificar elementos naturales desde niños. Alambique: Didáctica de las Ciencias Experimentales, 84, pp.55-60

MESTRES, A. y TORRES, M. (2008). Algunas pautas para la elaboración de claves dicotómicas y árboles de clasificación. Departamento de Didácticas Especiales. Facultad de Formación del Profesorado. Universidad de Las Palmas de GC.

PEQUETIEMPO (2012). ¿Es la araña un insecto? - El rap de los arácnidos”. Canal de youtube Pequetiempo: https://www.youtube.com/watch?v=YGZ0rhkAK04\&t=3s. 
PRO, A. (2013). Enseñar procedimientos: por qué y para qué. Alambique: Didáctica de las Ciencias Experimentales, 73, pp.69-76.

RIBERA, I., MELIC, A. y TORRALBA, A. (2015). Introducción y guía visual de los artrópodos. Revista IDE@-SEA, 2, pp.1-30.

TORRALBA-BURRIAL, A., ARIAS RODRÍGUEZ, A Y HERRERO, M. (2017). Generación de una colección virtual como recurso didáctico para la enseñanza-aprendizaje de la fauna en distintos grados universitarios. III Congreso Virtual Internacional de Educación, Innovación y TIC.

VILCHES, A. M., LEGARRALDE, T. I. y BERASAIN, G. (2012) Elaboración y uso de claves dicotómicas en las clases de biología. III Jornadas de Enseñanza e Investigación Educativa en el campo de las Ciencias Exactas y Naturales, La Plata, Argentina. En Memoria Académica. 